\title{
Elective freeze-all embryos: What is the scientific evidence?
}

\section{Gyun-Ho Jeon}

Department of Obstetrics and Gynecology, Haeundae Paik Hospital, Inje University College of Medicine, Busan, South Korea

\section{REVIEW}

Please cite this paper as: Jeon GH. Elective Freeze-All Embryos: What is the scientific evidence? AMJ 2017;10(11):921-926.

https://doi.org/10.21767/AMJ.2017.3173

\section{Corresponding Author:}

Gyun-Ho Jeon, M.D

Department of Obstetrics and Gynecology

University of Inje College of Medicine

Haeundae Paik Hospital

Haeundae-ro 875, Haeundae-gu, Busan, South Korea

Email: jeon285@hotmail.com

\section{ABSTRACT}

\section{Background}

With the advancement in cryopreservation techniques, studies on freezing all survived embryos and frozen-thawed embryo transfer (FTET) in the next cycle have been conducted in assisted reproduction.

\section{Aim}

To review the theoretical backgrounds and clinical outcomes of freeze all embryos, followed by FTET, compared with conventional fresh embryo transfer (FET), and suggest future research direction for policy on this issue.

\section{Methods}

A through literature search comparing clinical outcomes of FTET and FET was conducted in PubMed. The following keywords were used: 'frozen-thawed embryo transfer', 'fresh embryo transfer', 'cryopreserved-thawed embryo transfer', 'freeze all policy' from their inceptions through July 2017.

\section{Results}

Clinical researches conducted before 2013 indicated better in vitro fertilization (IVF) results as well as obstetric and perinatal outcomes with FTET than with FET. However, the 2017 Cochrane systemic review including more recent studies showed no difference in the cumulative live birth rate per woman between the two methods. FTET was associated with fewer miscarriages and a higher birth weight of singleton babies, but also with an increased rate of pregnancy complications such as preeclampsia.

\section{Conclusions}

While theoretical evidence of FTET after electively freezing all embryos has been suggested to have good outcomes through basic and clinical research, more high-quality randomized clinical trial results with the same primary outcomes under the well-controlled conditions are needed in the future for the generalization of the elective freeze-all method.

\section{Key Words}

Cryopreservation, freeze all policy, frozen-thawed embryo transfer

\section{What this review adds:}

\section{What is known about this subject?}

Several studies have suggested that FTET after electively freezing all embryos leads to better IVF results compared to FET.

\section{What new information is offered in this review?}

Recent studies including the 2017 Cochrane systemic review indicated no difference in the cumulative live birth rate per woman between FTET and FET.

3. What are the implications for research, policy, or practice?

More high-quality randomized clinical trial results with the same primary outcomes and well-controlled conditions are needed in the future for the generalization of the elective freeze-all method. 


\section{Background}

Pregnancy and live birth rates from assisted reproduction techniques including in vitro fertilization (IVF) have gradually increased. Furthermore, pregnancy using cryopreserved embryos has also increased since the first infant was born as a result of the frozen-thawed embryo transfer (FTET) in 1984. ${ }^{1}$ Traditionally, fresh embryo transfer (FET) following controlled ovarian hyperstimulation (COH) and IVF has been considered routine. $\mathrm{COH}$ is an important process for increasing pregnancy rates by inducing multiple follicles development in assisted reproduction. Interests in FTET without FET in the $\mathrm{COH}$ cycle have increased recently because of concerns regarding the negative effects of $\mathrm{COH}$ not only on the endometrium and uterine environments during implantation but also on the safety of the pregnancy and birthing process. ${ }^{2}$

Several studies have reported that pregnancy rates are decreased in FET cycles compared to FTET, owing to impaired endometrial receptivity and endometrium transformation immediately after $\mathrm{COH}$; others have also suggested that the obstetric and perinatal outcomes were poor in FET cycles. ${ }^{3,4}$ With the advancement in cryopreservation techniques, recent research has demonstrated the negative effect of $\mathrm{COH}$ and supraphysiological hormone levels on the endometrium, as well as the reduced risk of developing ovarian hyperstimulation syndrome (OHSS) by freezing all survived embryos and FTET in the next cycle. Although evidence of FTET after electively freezing all embryos has been suggested to have good outcomes through basic and clinical research, the results of recent clinical studies still challenge the scientific dogma. ${ }^{3,5,6}$

\section{Methods}

A through literature search was conducted in PubMed to gather the published articles comparing clinical outcomes of FTET and FET. The following keywords were used: 'frozenthawed embryo transfer', 'fresh embryo transfer', 'cryopreserved-thawed embryo transfer', 'freeze all policy', from their inceptions through July 2017. The references of the searched articles were also examined in an attempt to ensure that no important articles were inadvertently excluded. The inclusion criteria were all studies that were published upon comparing clinical outcomes of FTET and FET on humans and the exclusion criteria were all other papers performed on the animals and in vitro. In addition, some articles considered to have no direct relevance to the subject or with similar conclusions were excluded.
The effects of $\mathrm{COH}$ on the endometrial receptivity and uterine environments

\section{1) $\mathrm{COH}$ and genes related to implantation}

The endometrium is structurally and functionally matched to embryo implantation during the window of implantation, and many related genes have been studied. Recently, 179 genes associated with endometrial receptivity were selected from a database of 19,285 gene in the human endometrium, and it was proven that the expression of cell adhesion proteins (CD36, THBS1, and COMP) related to these genes significantly increased in the receptive phase of the endometrium. ${ }^{7}$ Recently, studies have shown that $\mathrm{COH}$ can alter the expression of these genes involved in the implantation. In one study on an oocyte donation program in $2005,^{8}$ the endometrium was sampled and gene expression was analyzed, comparing the fertile period on the $7^{\text {th }}$ day after the LH-surge (LH7) in the natural cycle and on the $7^{\text {th }}$ day after hCG-triggering injection (hCG7) after ovulation stimulation in the next menstrual cycle. In the latter samples, 558 gene expression patterns were altered compared with those in natural cycles; of these, 351 overlapped with the window of implantation-associated genes, which had changed expression on the LH7 when compared to the second day after LH-surge, the prereceptive phase. Among the 351 genes, the number of genes that increased on the LH7 and the hCG7 was very small; on the contrary, no genes that had decreased expression on the LH7, also had decreased expression on the hCG7, indicating that the gene expression on the hCG7 showed a tendency to be opposite to that on the LH7. In other words, the gene expression patterns during the normal implantable period tended to be reversed in many phases in the superovulation cycle.

\section{2) $\mathrm{COH}$ and endometrial receptivity and development}

Estradiol and progesterone concentrations following $\mathrm{COH}$ are significantly higher than in natural cycles due to injections of gonadotropin during $\mathrm{COH}$ (E2: $3.50 \pm 3.27$ vs. $0.15 \pm 0.11$ (nmol/L), P4: $5.63 \pm 2.74$ vs. $3.38 \pm 1.35$ (nmol/L). ${ }^{9}$ Because estradiol and progesterone in the blood are closely related to the development and maturation of the endometrium, their excessive concentrations are known to lead to the development and progression of the endometrium, the maturation of pinopode expression, and down-regulation of steroid receptor expression. ${ }^{10}$ This results in embryo-endometrial asynchrony, which is important at implantation and can lead to an increase in implantation failure. ${ }^{11}$ In 2004, Mirkin et al. compared the concentrations of estradiol and progesterone in the blood, histological progression of the endometrium, pinopode expression and maturation, and expression of steroid 
receptors between the natural and $\mathrm{COH}$ cycles. ${ }^{12}$ They found supraphysiologic and significantly higher estradiol and progesterone levels in the $\mathrm{COH}$ cycles than in the natural cycles (E2: $3003 \pm 981$ vs. $296 \pm 64$ (pg/mL), P4: $1.2 \pm 0.1$ vs. $0.9 \pm 0.1(\mathrm{ng} / \mathrm{mL})$ on the day of urinary LH surge or hCG administration). Additionally, they reported that advanced histological dating ( $\mathrm{H}-\mathrm{E})$ and pinopode maturation (regressing-disappearing characteristics), and accelerated estrogen and progesterone receptor down-regulation in the endometrium were observed in all $\mathrm{COH}$ cycles regardless of $\mathrm{COH}$ protocols, compared to those in the natural cycles (all $p<0.05)$

3) Increased serum progesterone concentration and endometrial advancement, pregnancy rate

As reported by Ubaldi et al. in 1997, early elevation of serum progesterone in the late follicular phase following $\mathrm{COH}$ is a major cause of endometrial maturation and early progression; moreover, this may result in decreased endometrial receptivity and embryo-endometrial asynchrony, which in turn may result in implantation failure and poor pregnancy rates. ${ }^{13}$ Since then, several studies related to this have been published. Ubaldi et al. performed an endometrial tissue aspiration test in cohorts with a serum progesterone level above $1.0 \mathrm{ng} / \mathrm{mL}$ and below $1.0 \mathrm{ng} / \mathrm{mL}$ on the day of hCG administration; as a control group, they assigned patients with normal serum progesterone concentration, who did not undergo endometrial tissue aspiration. They compared the implantation rates, clinical pregnancy rate, miscarriage rate, and early progression on endometrial biopsy among these groups. Patients with serum progesterone levels above $1.0 \mathrm{ng} / \mathrm{mL}$ showed a decreasing tendency, although not statistically significant, to have failed implantation, a lower clinical pregnancy rate, and a higher degree of progression of endometrial tissue compared with those in the other groups, indicating that a subtle increase of blood progesterone level could further promote the maturation of the endometrium. There was no case of pregnancy in patients where endometrial maturity was advanced more than 3 days; the same was found in another study in $2002 .{ }^{11}$ Since then, there have been no reported cases of successful pregnancy by FET if the progression of the endometrium advanced more than 3 days, suggesting that the decrease in endometrial receptivity due to the maturation and early progression of the endometrium after $\mathrm{COH}$ has a significant effect on the pregnancy rate. In another recent study of 3,296 cycles of IVF, ${ }^{14}$ a multifactorial analysis showed that the live birth rate was also significantly lower when the serum progesterone concentration was raised to $1.5 \mathrm{ng} / \mathrm{mL}$ on the day of hCG administration. This also suggests the decreased implantation potential with supraphysiologic progesterone levels after $\mathrm{COH}$, and there is contention as to what the cut-off values are.

\section{Comparison of in vitro fertilization results between FET and FTET}

A meta-analysis and systematic review published by Roque et al. in 2013 revealed only 3 randomized clinical trials that can be included in the meta-analysis among studies comparing FET and embryo transfer after elective freeze. ${ }^{3}$ The meta-analysis of 633 cycles in women 27-33 years of age showed that the ongoing pregnancy rate was improved by 32 per cent and the clinical pregnancy rate by 31 per cent using FTET; the miscarriage rate was not significantly different between the two groups. However, one of three studies included in this meta-analysis was retracted due to methodological problems; they then performed a new meta-analysis excluding the recalled study. The new analysis included 259 IVF cycles with blastocyst embryo transfers in normal and high responders; nonetheless, they found that FTET had a 31 per cent higher clinical pregnancy rate than FET. ${ }^{5}$ However, in the 2017 Cochrane systemic review, which included four randomized clinical trials comparing a freeze-all strategy with conventional IVF/ICSI strategy with FET, there was no clear evidence of a difference in cumulative live birth rate between two strategies (OR 1.09, 95 per cent confidence interval (Cl) 0.91-1.31; 1892 women; $\mathrm{I}^{2}=0$ per cent; moderate-quality evidence); however, time to pregnancy was not reported in any study. Additionally, they suggested that FTET lowered the OHSS risk for at-risk women with low-quality evidence (OR 0.24, 95 per cent $\mathrm{Cl} 0.15-0.38 ; 1633$ women; $\mathrm{I}^{2}=0$ per cent; lowquality evidence). ${ }^{6}$ The discrepancy in results between these two meta-analyses is attributed to the difference in primary outcome (Roque et al. report live birth rate per first transfer, whereas Wong et al. report cumulative live birth rate per woman). The authors of the Cochrane review contend that the live birth rate per first transfer is less relevant since at the same time of the first transfer in a freeze-all strategy, patients with a conventional strategy including the fresh transfer have already received the second transfer (in cases with a sufficient number of embryos).

These meta-analyses had some limitations. No method to directly measure the endometrial receptivity exists, as endometrial receptivity can only be deduced from the pregnancy rate results of several studies included in these meta-analyses. Moreover, these studies analyzed the results of only normal and high-responder groups, and there is a possibility that the quality of the endometrium was overestimated in FTET since the best embryos may have 
been selected during the embryo freezing and thawing process. Additionally, these studies had serious risk of bias associated with varying protocols for the endometrial preparation and freezing technique, unclear blinding of investigators, unit of analysis error, and absence of adequate study termination rules, which resulted in a judgement of the evidence as moderate to low quality. ${ }^{3,5}$

\section{Obstetric and perinatal outcome after FET and FTET}

A recent systematic review and meta-analysis showed that the risk of obstetric and perinatal complications is higher in singleton pregnancies by in vitro fertilization than in natural pregnancies. ${ }^{15}$ Whilst pregnancies following IVF may be at higher risk of complications, FTET has been associated with a lower burden of complications compared to FET. ${ }^{16}$ A metaanalysis and systematic review of the literature published in 2012 indicated that the risk of obstetric and perinatal complications was reduced in the FTET group (antenatal bleeding [RR=0.67, 95 per cent $\mathrm{Cl} 0.55-0.81]$, preterm birth [RR=0.84, 95 per cent $\mathrm{Cl} 0.78-0.90]$, small for gestational age [RR=0.45, 95 per cent $\mathrm{Cl} 0.30-0.66]$, low birth weight infant $[R R=0.69,95$ per cent $\mathrm{Cl} 0.62-0.76]$, perinatal mortality rate $[R R=0.68,95$ per cent $\mathrm{Cl} 0.48-0.96]) .{ }^{4}$ These results are also presumed to be caused by altered placentation related to supraphysiologic hormonal levels during $\mathrm{COH}$ in FET. They reported that, concerning the incidence of other major congenital anomalies, there was no difference between the FTET and FET groups. Spijker et al. also showed the risk of high birth weight $(>4,500 \mathrm{~g})$ and being large for gestational age was higher in the FTET group than in the FET group. ${ }^{17}$ However, there were some studies reporting no difference of live birth weight between fetuses after FTET and FET. ${ }^{18,19}$

Some results of studies related to the perinatal/obstetric outcome may be inconsistent, and they are mainly observational studies, thus it is considered that more randomized controlled studies are needed in the future. According to the 2017 Cochrane systemic review, FTET was associated with fewer miscarriages, a higher birth weight of singleton babies, and an increased rate of pregnancy complications. $^{6}$ The Cochrane review included only two studies in the analysis of pregnancy complications, and the increased rate of pregnancy complications in the FTET group corroborates the findings of a recent randomized study by Chen et al. in $2016 .{ }^{20}$ Chen et al. reported that the FTET group had a higher frequency of preeclampsia, but there were no significant differences in the rates of other pregnancy and neonatal complications between the FTET and FET groups.

\section{Other effects of FTET}

The most important aspect of OHSS management, which is one of the major complications of $\mathrm{COH}$, is prevention. The most effective preventive method is the replacement of hCG for final oocyte maturation and induction of ovulation, instead of preferencing GnRH-agonists which can reduce early-onset ovarian hyperstimulation by decreasing the expression of vascular endothelial growth factor (VEGF) and VEGF receptor 2 (VEGFR-2) mRNA. ${ }^{21}$ It is also known that methods of cryopreservation of all embryos and subsequent thawing and transfer at a later period (freeze-all strategy) can reduce delayed OHSS. $^{3}$ Devroey et al. have disseminated the OHSS-free clinic concept without OHSS through these two methods, and it is thought that most early and delayed OHSS can be prevented. ${ }^{22}$ In addition, recent studies have used a 24-chromosome screening test at the blastocyst stage, which showed higher implantation and pregnancy rates than FISH assay in the pre-implantation genetic test. ${ }^{23}$ Therefore, the test using the blastocyst stage in the pre-implantation genetic screening has been increased. The method of cryopreservation of all embryos and subsequent transfer (elective freeze-all) may also be useful in cases where it is difficult to obtain the results of the pre-implantation genetic tests on the 5th and 6th days of fertilization within the window of period for implantation, or when a discrepancy between fresh embryos and the endometrium for implantation is expected.

\section{Discussion}

There are limitations in deriving conclusions from this review of various studies that have not been controlled by a variety of factors that may affect ART outcomes. For example, patients' ovarian reserve, stage and grade of embryos transferred, freezing methods, protocols of ovarian stimulation and endometrial preparation should be consistent for the comparison of the clinical outcome between FTET and FET. Furthermore, more studies should be conducted in the fields of economic, social, and psychological cost-effectiveness of the cryopreserved embryo transfer, as well as technological methods for optimal embryo freezing, and established protocols for endometrial preparation. Additionally, there may be selection bias included, even if we try to review all the most recent relevant data. Nevertheless, this review can have a role to help understand the current status of FTET for the freeze-all policy based on the most recent meta-analyses and main articles.

\section{Conclusions}

While theoretical evidence of FTET after electively freezing all embryos has been suggested to have good outcomes, 
thus suggesting a new direction in assisted reproduction. However, the 2017 Cochrane systemic review showed no difference of cumulative live birth rate per woman; moreover, it showed an increased rate of pregnancy complications in FTET compared with FET. More highquality randomized clinical trials are needed, with homogenous primary outcomes under well-controlled conditions are needed in the future for the generalization of the elective freeze-all method.

\section{References}

1. Zeilmaker GH, Alberda AT, van Gent I, et al. Two pregnancies following transfer of intact frozen-thawed embryos. Fertil Steril. 1984:42:293-6.

2. Roque M. Freeze-all policy: is it time for that? J Assist Reprod Genet. 2015;32:171-6.

3. Roque M, Lattes K, Serra S, et al. Fresh embryo transfer versus frozen embryo transfer in in vitro fertilization cycles: a systematic review and meta-analysis. Fertil Steril. 2013;99:156-62.

4. Maheshwari A, Pandey S, Shetty A, et al. Obstetric and perinatal outcomes in singleton pregnancies resulting from the transfer of frozen thawed versus fresh embryos generated through in vitro fertilization treatment: a systematic review and meta-analysis. Fertil Steril. 2012;98:368-77.

5. Roque $M$, Valle $M$, Kostolias $A$, et al. Freeze-all cycle in reproductive medicine: current perspectives. JBRA Assist Reprod. 2017;21:49-53.

6. Wong KM, van Wely M, Mol F, et al. Fresh versus frozen embryo transfer assisted reproduction (Review). Cochrane Database Syste Rev. 2017;3:CD01184.

7. Bhagwat SR, Chandrashekar DS, Kakar R, et al. Endometrial receptivity: a revisit to functional genomics studies on human endometrium and creation of HGExERdb. PLoS One. 2013;8:e58419.

8. Horcajadas JA, Riesewijk A, Polman J, et al. Effect of controlled ovarian hyperstimulation in IVF on endometrial gene expression profiles. Mol Hum Reprod. 2005;11:195-205.

9. Humaidan $P$, Bungum $L$, Bungum $M$, et al. Reproductive outcome using a GnRH antagonist (cetrorelix) for luteolysis and follicular synchronization in poor responder IVF/ICSI patients treated with a flexible GnRH antagonist protocol. Reprod Biomed Online. 2005;11:679-84.

10. Develioglu OH, Hsiu JG, Nikas G, et al. Endometrial estrogen and progesterone receptor and pinopode expression in stimulated cycles of oocyte donors. Fertil Steril. 1999;71:1040-7.

11. Kolibianakis E, Bourgain C, Albano C, et al. Effect of ovarian stimulation with recombinant folliclestimulating hormone, gonadotropin releasing hormone antagonists, and human chorionic gonadotropin on endometrial maturation on the day of oocyte pick-up. Fertil Steril. 2002;78:1025-9.

12. Mirkin S, Nikas G, Hsiu JG, et al. Gene expression profiles and structural/functional features of the periimplantation endometrium in natural and gonadotropin-stimulated cycles. J Clin Endocrinol Metab. 2004;89:5742-52.

13. Ubldi F, Bourgain C, Tournaye $\mathrm{H}$, et al. Endometrial evaluation by aspiration biopsy on the day of oocyte retrieval in the embryo transfer cycles in patients with serum progesterone rise during the follicular phase. Fertil Steril. 1997;67:521-6.

14. Venetis CA, Kolibianakis EM, Bosdou JK, et al. Estimating the net effect of progesterone elevation on the day of hCG on live birth rates after IVF: a cohort analysis of 3296 IVF cycles. Hum Reprod. 2015;30:6849.

15. Pandey S, Shetty A, Hamilton $M$, et al. Obstetric and perinatal outcomes in singleton pregnancies resulting from IVF/ICSI: a systematic review and meta-analysis. Hum Reprod Update. 2012;18:485-503.

16. Healy DL, Breheny S, Halliday J, et al. Prevalence and risk factors for obstetric haemorrhage in 6730 singleton births after assisted reproductive technology in Victoria, Australia. Hum Reprod. 2010;25:265-74.

17. Spijkers S, Lens JW, Schats R, et al. Fresh and FrozenThawed Embryo Transfer Compared to Natural Conception: Differences in Perinatal Outcome. Gynecol Obstet Invest. 2017 May 13. [Epub ahead of print]

18. Galliano D, Garrido N, Serra-Serra V, et al. Difference in birth weight of consecutive sibling singletons is not found in oocyte donation when comparing fresh versus frozen embryo replacements. Fertil Steril. 2015;104:1411-8.

19. Aflatoonian A, Mansoori Moghaddam F, Mashayekhy $M$, et al. Comparison of early pregnancy and neonatal outcomes after frozen and fresh embryo transfer in ART cycles. J Assist Reprod Genet. 2010;27:695-700.

20. Chen ZJ, Shi Y, Sun Y, et al. Fresh versus frozen embryos for infertility in the polycystic ovary syndrome. N Engl J Med. 2016;375:523-33.

21. Gómez R, Soares SR, Busso C, et al. Physiology and pathology of ovarian hyperstimulation syndrome. Semin Reprod Med. 2010;28:448-57.

22. Devroey P, Polyzos NP, Blockeel C. An OHSS-Free Clinic by segmentation of IVF treatment. Hum Reprod. 2011;26: 2593-7.

23. Scott Jr RT, Franasiak JM, Forman EJ. Comprehensive 
chromosome screening with synchronous blastocyst transfer: time for a paradigm shift. Fertil Steril. 2014;102: 660-1.

\section{PEER REVIEW}

Not commissioned. Externally peer reviewed.

\section{CONFLICTS OF INTEREST}

The authors declare that they have no competing interests.

\section{FUNDING}

None 